\title{
A confiabilidade dos dados de mortalidadee morbidade por doenças crônicas não-transmissíveis
}

\author{
The accuracy of the official mortality and morbidity \\ statistics related to chronic non-communicable diseases
}

Ruy Laurenti 1

M aria Helena P. de M ello Jorge 1

Sabina Léa D. Gotlieb 1

\footnotetext{
1 Departamento de

Epidemiologia da Faculdade de Saúde Pública da

Universidade de São Paulo.

Av. Dr. Arnaldo 715 ,

Cerqueira César, 01246-904,

São Paulo SP.

laurenti@usp.br
}

\begin{abstract}
M ortality statistics are an important tool for the knowledge of the epidemiological profile of a population and, therefore, for planning actions in the health area. In Brazil, they are elaborated since 1975 by the M ortality Information System of the M inistry of $\mathrm{H}$ ealth, and has an estimated coverage of $82 \%$, varying through out the country. Concerning the accuracy of data, it is in course a gradual improvement, but there are, still, about $14 \%$ of deaths classified as ill defined. The objective of this paper is to show the accuracy of mortality and morbidity statistics related to chronic non-communicable diseases. Critics and commentaries are done, based on national and international investigations. It was possible to conclude that although they are not completely exact or accurate, these statistics are very relevant for epidemiological evaluations. It is important to say that the Brazilian mortality statistics, by the quality point of view, presents the similar accuracy then any other country with a long tradition in the area of health statistics.
\end{abstract}

Key words M ortality statistics, Accuracy, M orbidity statistics
Resumo As estatísticas de mortalidade constituem-se em importante subsídio para o conhecimento do perfil epidemiológico de uma população, elaboração de indicadores de saúde e conseqüente planejamento de ações desse setor. No Brasil, esses dados são rotineiramente elaborados, desde 1975, pelo Sistema de Informações sobre M ortalidade do M inistério da Saúde, que tem cobertura estimada em torno de $82 \%$, com variações nas regi ões do país. Quanto à confiabilidade dos dados, está ocorrendo uma melhora gradativa, mas há, ainda, cerca de $14 \%$ de mortes classificadas como mal definidas. 0 objetivo deste trabalho é mostrar a confiabilidade dos dados de mortalidade e morbidade por doenças crônicas não- transmissíveis. A partir de investigações levadas a efeito por pesquisadores nacionais e internacionais, são feitos comentários e críticas, sendo possível concluir que, embora ainda não totalmente exatos ou confiáveis, esses dados são relevantes para numerosas avaliações epidemiológicas. É bom ressaltar que os dados brasileiros de mortalidade, do ponto de vista qualitativo, têm exatidão e fidedignidade semelhantes aos de qualquer outro país de longa tradição na elaboração dessas estatísticas.

Palavras-chave Estatísticas de mortalidade, Estatísticas de morbidade, Confiabilidade 


\section{Introdução}

A primeira pessoa que elaborou e publicou estatísticas de mortalidade por causas foi John Graunt, em 1662, e o fato mais interessante é que, nessa obra, 0 autor já fazia comentários sobre a qualidade dos dados (Graunt, 1964).

Relativamente à exatidão dos dados de mortalidade, Rothman (1996) descreve os comentários de Graunt, que incluíam críticas aos métodos de registro e coleta das listas de mortalidade ("Bills of mortality") e a dificuldade em indicar o verdadeiro diagnóstico da causa de morte. Graunt apontava que, em al guns casos, essa indicação era fácil, como nas causas externas, porém bastante difícil, em muitos outros.

Embora com críticas, desde seu início, as estatísticas de mortalidade sempre foram e continuam sendo a principal fonte de dados para se conhecer o perfil epidemiológico de uma área, analisar tendências, indicar prioridades, avaliar programas, entre outras finalidades. E a experiência tem mostrado que, apesar de não serem ainda totalmente corretas, as estatísticas de mortalidade têm sido de grande utilidade. M oriyama (1979) descreve e comenta os usos dos dados de mortalidade, referindo que eles serviram às necessidades da saúde pública, durante longo período de tempo, quando o foco principal era o saneamento e 0 controle das doenças infecciosas. Com o declínio da incidência das mesmas, houve, gradualmente, aumento das doenças crônicas nãotransmissíveis, com o conseqüente interesse voltado para elas. Nessas condições, muitos passaram a considerar que os dados de mortalidade não serviam para caracterizar essa transição, por várias razões. Uma delas a de que, freqüentemente, é difícil determinar a causa básica de morte para as doenças crônicas; outra razão apontada, a tradicional seleção de uma única causa de morte leva à perda de informações úteis sobre doenças concomitantes; e sabe-se, por outro lado, que os idosos acumulam várias doenças crônicas não-transmissíveis. Há ainda críticas de que as doenças crônicas de baixa letalidade, praticamente, não aparecem nas estatísticas de mortalidade.

Ainda segundo Moriyama (1979), além do aumento da insatisfação em relação às estatísticas de mortalidade, existia um sentimento crescente de que eram necessárias as estatísticas de morbidade, para os propósitos da saúde pública (... there emerged a feeling that it was morbidity and not mortality statistics that was need for public health purposes). Pelo menos nos Estados Unidos, a demanda por estatísticas de morbidade cresceu e culminou na aprovação do Health Survey Act, em 1955, o qual iria produzir estatísticas de morbidade e incapacidades, em nível nacional. Assim, iniciou-se 0 H ealth Interview Survey, que coletava dados sobre "sentir-se doente" ou "não se sentir bem" (illness) e "incapacidades", não se obtendo dados de doenças ou diagnósticos propriamente ditos que, para al gumas doenças específicas (exemplo: hipertensão arterial, diabetes), eram conseguidos por meio de exames médicos de uma subamostra.

A necessidade de dados de "doenças" ou "diagnósticos" para a população geral, bem como para pequenas áreas, renovou o interesse pelas estatísticas de mortalidade. Aumentou, acentuadamente, também, a preocupação com os efeitos de problemas ambientais sobre a saúde. A poluição ambiental, as radiações ionizantes, a contaminação química e os efeitos sobre a saúde levaram à necessidade de estudos quantitativos e, dessa maneira, houve aumento do número de estudos epidemiológicos, particularmente a partir da segunda metade do século 20. É de se notar que a maioria desses estudos era baseada em dados de mortalidade. Assim, as estatísticas de mortalidade continuaram bastante utilizadas no mundo, apesar de todas as críticas que a elas vêm sendo feitas. Quanto às estatísticas de morbidade, isto é, para doenças ou diagnósticos, elas são desejáveis, mas obtê-las de maneira rotineira nem sempreé fácil.

No Brasil, os dados de mortalidade são obtidos a partir do Sistema de Informações sobre M ortalidade (SIM ). Para os de morbidade, além daqueles advindos das doenças de notificação obrigatória, das doenças e acidentes de trabalho, existem as estatísticas sobre saídas hospitalares, que incluem os diagnósticos e estão no Sistema de Informações Hospitalares do SIH-SUS.

\section{A confiabilidade dos dados de mortalidade}

A análise da exatidão dos dados de mortalidade por causas, de uma maneira geral, e das doenças crônico-degenerativas, em especial, pode ser apresentada, de forma resumida e didática, por meio de proporções de óbitos por 
"causas mal definidas" e por "diagnósticos incompletos" e de estudos especiais.

\section{Proporções de óbitos por "causas mal definidas" e por "diagnósticos incompletos"}

Tradicionalmente, a proporção de "causas mal definidas" tem sido utilizada para avaliar a qualidade dos dados de mortalidade por causas. Entende-se como causas mal definidas aquelas que são declaradas como "sintomas", "sinais", ou pelas expressões "causa indeterminada", "causa ignorada", "sem assistência médica" e outras similares.

Sabe-se que, quanto maior a proporção de óbitos por causas mal definidas em uma distribuição, menor éa exatidão dessas estatísticas. É de se lembrar também que, praticamente, não existem dados de mortalidade por causas sem a presença de mal definidas, porém, em elevado número de vezes, esta proporção é baixa, aceitando-se, como tal, aquela inferior a $4 \%-6 \%$. De uma maneira geral, estes valores são observados nas áreas ou países mais desenvolvidos, diferentemente do que ocorre naqueles menos desenvolvidos. Já foi, até mesmo, proposto que a proporção de "mal definidas" fosse considerada um indicador do nível de saúde e de desenvolvimento de uma região.

A porcentagem de óbitos por causas mal definidas para o Brasil é alta, porém, há grande variabilidade entre os Estados (Tabela 1), sendo, entretanto, essa proporção, bastante baixa nas capitais. Chama a atenção o valor apresentado pelo Piauí ( $30,7 \%)$, enquanto na capital desse Estado a proporção encontrada foi de $3,4 \%$.
Para áreas onde a proporção de causas mal definidas é alta, tem sido proposto que se faça uma redistribuição desses casos segundo a proporção dos óbitos em cada um dos capítulos de causas bem definidas da Classificação Internacional de Doenças - CID (OM S, 1995). Essa metodologia tem sido discutida e outras vêm sendo propostas (M ello Jorge et al., 2002). Estes autores, visando estudar o problema das causas mal definidas, realizaram investigação em 13 municípios brasileiros, em um total de 979 mortes, das quais, 294 (30\%) pertenciam a essa categoria. Para cada óbito foi feita revisão de prontuário médico hospitalar, entrevistas com médicos e, em muitos casos, entrevista no domicílio com os familiares do falecido. Foi possível esclarecer os diagnósticos em $71,1 \%$ dos casos. Verificou-se que $25,8 \%$ e $9 \%$, respectivamente, eram doenças do aparelho circulatório e neoplasias, o que não diferiu muito da distribuição original; entretanto, al gumas causas aumentaram acentuadamente, como por exemplo os transtornos mentais e comportamentais.

Outra pesquisa, que estudou os óbitos de mulheres de 10 a 49 anos nas capitais de Estados brasileiros, mostrou que, em $8,1 \%$ dos atestados de óbito originais, a causa preenchida era mal definida. A investigação possibilitou esclarecer total mente cerca de $65 \%$ dos casos, restando entretanto ainda 2,9\% de mal definidas. Vários desses óbitos eram de mulheres que tiveram assistência médica e internação em hospital, mas não foi possível chegar a um diagnóstico e não havia sido feita necropsia. Nesse estudo, a distribuição dos diagnósticos, após os esclarecimentos, não foi semelhante à distribuição original (Laurenti et al., 2002).

\section{Tabela 1}

Ó bitos totais e por causas mal definidas (№ e \%) no Brasil, Estados de São Paulo, Rio Grande do Sul, Piauí e respectivas capitais, 2000.

\begin{tabular}{lcrc}
\hline Área & Óbitos totais & \multicolumn{2}{c}{ Óbitos mal definidos } \\
& No & No & $\%$ \\
\hline Brasil & 946.392 & 135.645 & 14,3 \\
São Paulo (Estado) & 238.959 & 15.725 & 6,6 \\
São Paulo (Município) & 68.326 & 792 & 1,2 \\
Rio Grande do Sul & 67.953 & 2.993 & 4,4 \\
Porto Alegre & 9.859 & 106 & 1,1 \\
Piauí & 11.623 & 3.566 & 30,7 \\
Teresina & 3.477 & 119 & 3,4 \\
\hline
\end{tabular}

Fonte: SIM /M S (dados brutos) 
Além dessas causas, outro tipo de diagnóstico, muitas vezes declarado pelos médicos nos atestados de óbito, é representado pelas causas ou diagnósticos "incompletos". São causas básicas declaradas apenas como "cardiopatia", "doença do coração", "nefropatia”, "hepatopatia", "neoplasia", "câncer" e outras, sem haver especificação do tipo da doença. No caso das doenças infecciosas, isso ocorre, por exemplo, com a "septicemia", que, quando selecionada como causa básica, é um diagnóstico incompleto, visto ser sempre consequêencia ou complicação de outro agravo.

$\mathrm{Na}$ tabela 2, pode ser apreciada a situação relativa aos diagnósticos incompletos dos dois princi pais conjuntos de causas de morte, doenças do aparelho circulatório e neoplasias, ambas, doenças crônicas não- transmissíveis. No caso de doenças do aparelho circulatório, incluem-se as arritmias cardíacas, bloqueios atrioventriculares e outros bloqueios e taquicardias ( códigos I44 a I 49 da CID-10), bem como os diagnósticos insuficiência cardíaca e insuficiência cardíaca congestiva (códigos I50 e 151 da CID-10). No caso das neoplasias, são considerados diagnósticos incompletos os códigos C76 a C80 da CID-10, neoplasia maligna de localizações mal-definidas, secundária e de localizações não especificadas.

Verifica-se que, para o Brasil, $14,2 \%$ das doenças do aparelho circulatório informadas como causa de morte correspondem a diagnósticos incompletos, isto é, não especificam qual a doença responsável pelo óbito. 0 mesmo ocorre em $8,1 \%, 9,4 \%$ e $12,3 \%$, respectivamente, para
São Paulo, Porto Alegre e Teresina. Os diagnósticos incompletos, nos casos de morte por neoplasias, apresentam-se em menor proporção do que as observadas nos casos de doenças do aparelho circulatório. Pode-se dizer, portanto, que a informação de mortalidade por neoplasia é um pouco mais confiável no que se refere à identificação de tipos específicos (Tabela 2).

Pesquisa realizada para verificar, a partir dos óbitos com diagnósticos incompletos, quais seriam as verdadeiras causas básicas mostrou que, esclarecido o diagnóstico, em quase todos os casos, este permanecia no mesmo grupo de doenças (capítulo da CID-10). Assim, entre 181 óbitos com diagnóstico incompleto nos atestados originais, observaram-se 39 casos de doenças do aparelho circulatório e, após investigação, nove casos migraram para outros capítulos. Sete casos que estavam como "incompletos" em outros capítulos foram incluídos como doenças do aparelho circulatório. Dessa forma, o número final, em vez de 39, foi de 35 (para dois casos não se encontraram os prontuários). Com os diagnósticos incompletos de neoplasias verificou-se que, dos 10 casos, 2 não eram; por outro lado, 6 eram neoplasias, mas estavam em outros capítulos. Portanto, os 10 casos iniciais "incompletos" tornaram-se 14 casos de neoplasias (M ello Jorge et al., 2002).

Fica claro que os diagnósticos incompletos prejudicam a confiabilidade dos dados para causas específicas, mas são responsáveis por alterações muito pequenas, quando as estatísticas de mortalidade por causas são apresentadas segundo grupos afins ou capítulos da CID.

\section{Tabela 2}

Óbitos por doenças do aparelho circulatório (I00-199, CID-10), neoplasias (C00-C97, D00-D 48) e seus respectivos diagnósticos incompletos. Brasil, Estados de São Paulo, Rio Grande do Sul, Piauí e capitais, 2000.

\begin{tabular}{|c|c|c|c|c|c|c|c|c|c|c|c|}
\hline \multirow[t]{3}{*}{ Área } & \multirow{3}{*}{$\begin{array}{l}\text { Óbitos } \\
\text { Totais }\end{array}$} & \multicolumn{5}{|c|}{ Doenças do aparelho circulatório } & \multicolumn{5}{|c|}{ Neoplasias } \\
\hline & & \multicolumn{2}{|c|}{ Total } & \multicolumn{3}{|c|}{ Diagnósticos incompletos } & \multicolumn{2}{|c|}{ Total } & \multicolumn{3}{|c|}{ Diagnósticos incompletos } \\
\hline & & no & $\%$ & no & $\%(1)$ & $\%(2)$ & no & $\%$ & no & $\%(1)$ & $\%(2)$ \\
\hline Brasil & 946.392 & 260.553 & 27,5 & 37.018 & 3,9 & 14,2 & 120.493 & 12,7 & 9.803 & 1,0 & 8,1 \\
\hline São Paulo (Estado) & 238.959 & 72.692 & 30,4 & 9.064 & 3,8 & 12,5 & 35.511 & 14,9 & 2.405 & 1,0 & 6,8 \\
\hline São Paulo (M unicípio) & 68.326 & 22.435 & 32,8 & 1.813 & 2,7 & 8,1 & 11.515 & 16,9 & 575 & 0,8 & 5,0 \\
\hline Rio Grande do Sul & 67.953 & 22.870 & 33,7 & 2.825 & 4,2 & 12,3 & 13.079 & 19,2 & 967 & 1,4 & 7,4 \\
\hline Porto Alegre & 9.859 & 3.260 & 33,1 & 305 & 3,1 & 9,4 & 2.122 & 21,5 & 117 & 1,2 & 5,5 \\
\hline Piauí & 11.623 & 2.728 & 23,5 & 428 & 3,7 & 15,7 & 873 & 7,5 & 65 & 0,6 & 7,5 \\
\hline Teresina & 3.477 & 1.075 & 30,9 & 132 & 3,8 & 12,3 & 422 & 12,1 & 27 & 0,8 & 6,4 \\
\hline
\end{tabular}

(1) \% em relação ao total de óbitos

(2) \% em relação ao total de óbitos por doenças do aparelho circulatório ou neoplasias 


\section{Estudos especiais para avaliar a confiabilidade dos dados de mortalidade}

Sobre a exatidão dos dados de mortalidade, são numerosos os trabalhos publicados, particularmente, a partir da segunda metade do século 20. No artigo "Accuracy of the statements of the cause of death on death certificates and the international comparability of mortality statistics", os autores discutem a qualidade dos dados com base em extensa bibliografia sobre o tema (Laurenti et al., 2000). 0 trabalho feito pelo Centro Colaborador da OM S para os Países Nórdicos "Suggested references on validity of cause of death" informa mais de 200 artigos publicados a esse respeito.

Vários são os estudos ou investigações especiais, com metodologias próprias, para avaliar a confiabilidade dos dados de mortalidade por causas.

\section{Atestados de óbito originais comparados com informações clínicas}

É possível avaliar a exatidão das informações declaradas pelos médicos no atestado de óbito original, comparando-o com o atestado refeito, baseado em dados contidos nos prontuários médicos, informações dadas por médicos, exames de laboratórios, laudos de autópsias etc. bem como, muitas vezes, entrevistas junto com familiares da pessoa falecida.

Há vários estudos, deste tipo, nacionais e internacionais. Um dos mais clássicos, "Patterns of Urban M ortality - Report of the InterAmerican Investigation of M ortality" (Puffer \& Griffith, 1968), avaliou, entre outros aspectos, a fidedignidade das informações sobre causas de morte em dez cidades da América Latina, uma dos Estados Unidos e outra da Inglaterra. Duas cidades brasileiras (São Paulo e Ribeirão Preto) foram incluídas e, embora o trabalho de campo tivesse sido realizado no período de 1962-1964 e a publicação date de 1968, no que se refere à metodologia, ele permanece bastante atual. Alguns resultados dessa investigação, para três doenças crônico-degenerativas (neoplasias malignas, doenças do aparelho circulatório e diabetes), são mostrados na tabela 3. Podem ser apreciadas, também, a comparação entre 0 atestado de óbito "original" e o "final", após a coleta de informações adicionais, e as "exclusões" e as "inclusões" em cada diagnóstico.
Esses resultados apresentam aspectos interessantes do ponto de vista da exatidão dos dados. Assim, no caso das mortes por neoplasias malignas, as diferenças entre 0 "final" e 0 "original" são mínimas e, desta maneira, poder-seia dizer que a mortalidade por esse grupo de causas é bastante fidedigna. Entretanto, ainda que haja razoável compensação entre "o que sai" e o "que entra", essa é apenas quantitativa e não qualitativa, o que quer dizer que idades e sexos poderão ser diferentes e, portanto, o perfil epidemiológico estará afetado. Também não há compensação entre al guns tipos de câncer e o exemplo mais notável é o do colo do útero, visto que houve somente inclusão, evidenciando que o mesmo estava "subinformado", nos dados oficiais.

Ainda na tabela 3 é curioso o observado com relação às mortes por doença isquêmica do coração em Ribeirão Preto, onde houve igualdade de valores entre 0 "original" e 0 "final", devido ao fato de as "exclusões" (26,5\% dos casos) serem equivalentes às "inclusões".

Em outra investigação recém-terminada, que estudou os óbitos de mulheres de 10 a 49 anos nas capitais brasileiras, os resultados mostraram que, com os dados corrigidos, as neoplasias aumentaram cerca de $6 \%$, as doenças do aparelho circulatório diminuíram $1 \%$ e as doenças endócrinas e metabólicas, com destaque para o diabetes, aumentaram em $52 \%$. Digno de nota foram os transtornos mentais e comportamentais que, em freqüência, cresceram $112 \%$ (estiveram presentes apenas em 0,5\% dos atestados originais e passaram a $1 \%$ nos atestados refeitos, a partir de informações adicionais). Assim, para mulheres desse grupo etário (10 a 49 anos), após a correção, a soma de algumas doenças crônicas não-transmissíveis, a saber, neoplasias, doenças do aparelho circulatório, doenças endócrinas e metabólicas e transtornos mentais e comportamentais representou $49,7 \%$ do total, contra $12,4 \%$ de doenças infecciosas e parasitárias (aproximadamente $61 \%$ delas eram Aids) e $15,5 \%$ de causas externas, com ênfase ao homicídio (Laurenti et al., 2002).

Com base nesse mesmo trabalho, estão apresentados dados para al gumas doenças crônicas não-transmissíveis, comparando-se 0 "original" com o "final" e as respectivas "inclusões" e"exclusões" (Tabela 4). 
Tabela 3

Número de óbitos segundo algumas causas básicas originais e finais, com as "inclusões" e "exclusões", após a pesquisa, em quatro cidades. Investigação Interamericana de M ortalidade de Adultos, 1962-1964.

\begin{tabular}{lcccccc}
\hline Cidade & Doenças & Original (0) & Exclusões & Inclusões & Final (F) & Razão (F/O) \\
\hline Bogotá (CO) & 1 & 632 & 137 & 159 & 654 & 1,03 \\
& 2 & 41 & - & 21 & 62 & 1,51 \\
& 3 & 1.226 & 489 & 446 & 1.183 & 0,96 \\
& 4 & 246 & 32 & 94 & 308 & 1,25 \\
\hline Bristol (RU) & 5 & 56 & 12 & 8 & 52 & 0,93 \\
& 1 & 1.043 & 120 & 196 & 1.119 & 1,07 \\
& 2 & 19 & - & 2 & 21 & 1,11 \\
& 3 & 2.072 & 479 & 370 & 1.963 & 0,95 \\
& 4 & 957 & 100 & 129 & 986 & 1,03 \\
\hline São Paulo & 5 & 36 & 10 & 20 & 46 & 1,28 \\
& 1 & 818 & 196 & 200 & 822 & 1,01 \\
& 2 & 63 & - & 7 & 70 & 1,11 \\
& 3 & 1.872 & 704 & 620 & 1.788 & 0,96 \\
& 4 & 499 & 80 & 122 & 541 & 1,08 \\
& 5 & 127 & 41 & 40 & 126 & 0,99 \\
\hline Ribeirão Preto & 1 & 165 & 35 & 49 & 179 & 1,08 \\
& 2 & 5 & - & 4 & 9 & 1,80 \\
& 3 & 331 & 126 & 138 & 343 & 1,04 \\
& 4 & 68 & 18 & 18 & 68 & 1,00 \\
& 5 & 18 & 5 & 10 & 23 & 1,28 \\
\hline
\end{tabular}

1= Neoplasias malignas / 2 = Câncer de colo de útero / 3= Doenças do aparelho circulatório /

$4=$ D oença isquêmica do coração / $5=$ Diabetes mellitus.

Fonte: Puffer \& Griffith, 1968

\section{Tabela 4}

Número de óbitos de mulheres de 10 a 49 anos segundo algumas doenças crônicas não-transmissíveis, nas declarações de óbito "original" e "final", exclusões e inclusões, após investigação. Capitais de estados brasileiros, 2002.

\begin{tabular}{lcccc}
\hline Causa & Original & Exclusões & Inclusões & Final \\
\hline Neoplasias & & & & \\
$\quad$ Mama & 390 & 2 & 35 & 423 \\
$\quad$ Colo do útero & 196 & 18 & 68 & 246 \\
Útero (não especificado) & 28 & 21 & 6 & 13 \\
$\quad$ Pulmão & 98 & 16 & 7 & 89 \\
D. Aparelho Circulatório & & & & \\
$\quad$ Acid. vascular cerebral & 569 & 94 & 102 & 577 \\
D. Isquêmica do coração & 337 & 149 & 90 & 278 \\
$\quad$ Insuficiência cardíaca & 47 & 35 & 29 & 41 \\
Diabetes & 162 & 22 & 148 & 288 \\
Causas Externas & & & & 318 \\
$\quad$ Acidentes transporte & 141 & - & 177 & 198 \\
Suicídios & 82 & - & 118 & 444 \\
Homicídios & 346 & 291 & 16 & 72 \\
$\quad$ Tipo ignorado & 347 & & & \\
\hline
\end{tabular}

Fonte: Laurenti et al., 2002 


\section{Causas de morte declaradas nos atestados de óbito comparadas com achados de necropsias}

Ao avaliar a exatidão dos dados de mortalidade, muitos autores consideram "padrão ouro" os diagnósticos obtidos nas necropsias. Ainda que estes se constituam em excelente fonte de informação para o preenchimento de causas nas declarações de óbito, sabe-se que não é bem assim. De fato, mesmo que $100 \%$ das mortes fossem submetidas à necropsia, não se teria $100 \%$ de exatidão nas mesmas, visto que nem todos os patologistas preenchem adequadamente os atestados de óbito. Há estudos clássicos, como o realizado, em 1900, por Cabot (1912) que, para 3.000 casos, comparou dados de necropsias com dados clínicos e a informação sobre causas de morte. Para o autor, quando a evolução para o óbito é muito rápida, a proporção de erros de diagnóstico égrande, como o verificado com a hemorragia cerebral ea oclusão coronária.

Em levantamento feito em Nova York e NovaJersey, na década de 1940, encontrou-se uma concordância de $96 \%$ para tuberculose pulmonar e $55 \%$ para miocardite crônica entre os atestados de óbito e os achados de necropsia (Pohlen \& Emerson, 1942). Na Inglaterra e Gales houve uma concordância geral da ordem de 45\% (Heasman \& Lipworth, 1966); para 0 Japão, Suécia e Tchecoslováquia, verificou-se que a hemorragia cerebral estava superestimada nas estatísticas nacionais com um conseqüente "déficit" de trombose e embolia cerebral (Kagan et al., 1967). Em Jerusalém, outro estudo mostrou que os atestados de óbito comparados aos achados de necropsias eram uma boa fonte de informação para neoplasias malignas; porém, havia limitações para os casos de doença do coração, doença cerebrovascular, embolia pulmonar, úlcera péptica e cirrose (A bramson et al., 1971).

Entre nós, há poucos estudos comparando as informaç̧ões do atestado de óbito com dados de autópsia. Uma investigação feita em hospital universitário mostrou que em $10,9 \%$ dos casos havia discordâncias entre essas duas fontes (Sanches, 2003).

Por outro lado, apenas os dados da necropsia não são, muitas vezes, suficientes para elaboração de uma seqüência causal lógica e adequado preenchimento da declaração de óbito. Pensa-se, nesse caso, haver necessidade também de informações clínicas, razão pela qual acredita-se que a melhor forma de preencher a declaração de óbito é a originada da união das informações de uma necropsia e um prontuário, ambos bem elaborados.

\section{Outros tipos de estudos para avaliar a exatidão dos dados de mortalidade}

Entre estes, podem ser citados os estudos de coorte, principalmente de dois tipos. 0 primeiro, aquele em que um grupo de indivíduos com a mesma doença é acompanhado no tempo e, ao ocorrer a morte, 0 atestado de óbito é analisado (Barros et al., 1985). Outro tipo é aquele em que a coorte é definida por uma característica comum, como por exemplo, pacientes atendidos no Hospital de Veteranos de Guerra do Vietnã. M oyer e colaboradores (1989) avaliaram as causas de morte declaradas nos atestados de óbito desses indivíduos e encontraram sensibilidade e especificidade maiores que $90 \%$ nos casos de acidente de trânsito, suicídios e homicídios, porém, mais baixas, em mortes por outras causas.

Outro tipo de metodologia é a que se baseia na comparação de atestados de óbito com os dados de Registros Especiais de D oenças, como por exemplo, o câncer (Breaux \& Perez, 1984; Rutqvist, 1985; e Demers et al., 1992). Trabalho deste tipo, realizado na Itália, concluiu que os dados de mortalidade para esclerose lateral amiotrófica eram bastante satisfatórios para estudos epidemiológicos (Chio et al., 1992).

Outro método, como a comparação de dois bancos de dados - um proveniente do sistema oficial de mortalidade e 0 outro originário de resumos de prontuários médicos (incluindo a causa da morte) de hospitais selecionados - , pode ser apontado. Em um estudo deste tipo encontrou-se concordância de $72 \%$ (Gittelsohn \& Senning, 1979) e, em outro, realizado na Espanha, valor igual a 96\% (Garrucho et al., 1990).

\section{A análise da mortalidade segundo causas múltiplas}

Foi também John Graunt, ao realizar e publicar o seu primeiro trabalho, quem estabeleceu que, para cada morte, apenas uma causa era indicada como a responsável. Entretanto, ele mesmo fez comentários sobre "morrer devido a uma doença" e "morrer com doenças". Neste item, de forma diversa dos anteriores, será adotada uma abordagem diferente para a confiabilidade e análise de causas de morte. 
Trata-se da chamada "metodologia das causas múltiplas", a qual não só enriquece, mas acrescenta informações, principalmente, nos casos de doenças crônicas não-transmissíveis.

Na metodologia das causas múltiplas, para cada caso de morte, seleciona-se a causa básica da maneira tradicional e verificam-se as doenças associadas, bem como as complicações da causa básica que estavam presentes. A importância desse método para as doenças crônicas não-transmissíveis é inconteste. De fato, particularmente nos mais idosos, são freqüentes associações bastante comuns, como por exemplo, as complicações da aterosclerose com a hipertensão arterial e com o diabetes mellitus.

No primeiro estudo realizado em nosso meio com essa metodologia, foi observado que, entre outros achados, na amostra de óbitos analisados, o diabetes estava presente, como causa básica, em 36 casos e, como causa múltipla, em 77, sendo que havia associação com hipertensão arterial, doença isquêmica do coração e acidente vascular cerebral, respectivamente, em $33,7 \%, 31,1 \%$ e $42,8 \%$ e com doenças das artérias, em $44,1 \%$. É de se ressaltar que a somatória das diferentes associações de duas ou mais causas pode ser maior que $100 \%$, visto que uma mesma causa pode se associar com várias outras (Laurenti, 1973). Na mesma amostra, verificou-se que a hipertensão arterial aparecia como causa básica em apenas 1,7\% dos casos e, como causa múltipla, em 10,8\%.

A hipertensão arterial é, talvez, a doença crônica não-transmissível mais freqüente nas populações adulta e idosa. A avaliação desta freqüência, por meio dos dados de mortalidade, não traduz a realidade, visto que, mesmo quando o médico declara essa causa de maneira correta no atestado de óbito, devido às regras internacionais de codificação da causa básica, na maioria das vezes, a hipertensão é descartada. Isso ocorre quando a hipertensão arterial está associada à doença isquêmica do coração e com os acidentes vasculares cerebrais pois estas causas têm preferência, por ocasião da codificação. Somente a "metodologia de causas múltiplas" de morte possibilita avaliar a freqüência da hipertensão arterial, pois ela vai aparecer como causa básica (em pequeno número) e causa associada (em um número muito maior).

No já referido estudo sobre mortes de mulheres de 10 a 49 anos nas capitais brasileiras (Laurenti et al., 2002), para um total de 7.332 óbitos analisados, a hipertensão apareceu como causa básica em 291 casos (3,9\%) e, co- mo causa associada, em 1.420 casos (19,4\%). Portanto, em um total de 1.711 menções, em apenas 291 foi selecionada como causa básica, isto é, da maneira como aparece nas estatísticas de mortalidade. I sso ocorreu porque, na maioria das vezes, ela estava associada com acidente vascular cerebral ou com doença isquêmica do coração e estas, pelas regras, tiveram prioridade, conforme já salientado.

0 diabetes mellitus também aparece como causa básica, somente, em uma pequena proporção das vezes em que é mencionado no atestado de óbito. Isto, entretanto, não é devido à questão das regras de seleção da causa básica, como no caso da hipertensão, mas ao fato de os médicos, quase sempre, declararem essa causa na Parte II do atestado de óbito, como causa contributória. Assim, uma boa caracterização epidemiológica de seu comportamento na mortalidade será sempre melhor quando realizada por análise de causas múltiplas.

No já citado estudo sobre análise da mortalidade por causas múltiplas (Laurenti, 1973), 0 diabetes apareceu como causa básica em 46,7\% das vezes em que foi mencionado na declaração de óbito. Para algumas outras doenças crônicas não-transmissíveis, verificou-se o seguinte: as avitaminoses e outras deficiências nutricionais, em 5,8\%; anemias, em 3,5\%; epilepsia, em $50,0 \%$; doença reumática crônica do coração, em $72,7 \%$; doença isquêmica do coração, em $58,3 \%$; acidentes vasculares cerebrais, em 58,3\%; trombose venosa e embolia, em 3,0\%; úlcera péptica, em $68,2 \%$; cirrose hepática, em 58,2\%; anomalia congênita do coração, em $75,6 \%$, entre outras. É preciso comentar que, nos exemplos aqui citados, as doenças são de claradas como causa básica ou não e a análise por causas múltiplas permite, como já comentado, mostrar a sua freqüência no momento da morte. Dessa maneira, enriquece muito a informação, melhorando, portanto, a caracterização do perfil epidemiológico da mortalidade por causas.

\section{A confiabilidade dos dados de morbidade}

No Brasil, os três principais sistemas de informações de morbidade são o referente às internações hospitalares (SIH/SU S), o de informações ambulatoriais (SIA/SUS) e o que trata dos agravos de notificação (SINAN). Entretanto, diferentemente do que ocorre com as informa- 
ções sobre mortalidade, praticamente, quase não existem estudos sobre a exatidão dos seus dados.

\section{Morbidade hospitalar}

O sistema conhecido como SIH/SUS coleta informações referentes às internações em hospitais próprios do SUS ou com ele conveniados e contém cerca de 11 a 12 milhões de internações por ano (Data SUS-SIH, 2002). Há várias críticas aos dados, podendo-se dizer, de uma maneira geral, que a abrangência do Sistema está limitada às internações no âmbito do SUS, excluindo-se, portanto, as custeadas diretamente ou cobertas por seguro-saúde (RIPSA, 2002). O utro problema refere-se ao fato de 0 mesmo paciente poder ser internado, pela mesma doença, mais de uma vez ao ano e, assim, seu diagnóstico aparecer repetido, o que vai resultar em contagem cumulativa. Talvez a maior crítica resida na questão da comparabilidade de seus dados, visto que não existe, no país, diferentemente do que ocorre com a mortalidade, um documento único padronizado para se declarar o diagnóstico de saída ou motivo da internação.

Tem sido observado que, entre nós, cada hospital ou mesmo cada médico tem critérios próprios para isso. Assim, como exemplo, suponha-se um paciente internado com insuficiência cardíaca e fibrilação auricular devidas a cardiopatia aterosclerótica; cada um desses diagnósticos pode ser declarado como o "principal". Além disso, às vezes, um diagnóstico é considerado principal quando foi o motivo de internação e, outras, aquele para o qual se teve o maior tempo de internação ou foram gastos mais recursos.

A partir da 10a Revisão da Classificação Internacional de Doenças (CID-10), está prevista a esperada definição recomendada para a codificação de causas na internação hospitalar: a afecção a ser usada em análise por causa única, em morbidade, é a afecção principal tratada ou investigada durante um episódio relevante de atenção à saúde. A afecção principal é definida como a afecção diagnosticada no final da consulta (internação), primariamente responsável pela necessidade de tratamento ou investigação do paciente (OMS, 1995 v. II). Entretanto essa definição, pelos motivos expostos, nem sempre é seguida.

Um estudo feito com 12.218 .632 saídas hospitalares, no ano de 1998, avaliou a existência de inconsistências quanto a: 1) tipo de doença ( por exemplo, aquelas inexistentes no País ou erradicadas); 2) diagnóstico e sexo; e 3) diagnóstico e idade. Os resultados mostraram, respectivamente, no primeiro grupo, 644 casos ou $0,0053 \%$ do total; no segundo, 17 casos ou $0,00014 \%$ e, no terceiro, 52.656 casos $(0,43 \%)$. 0 total de inconsistências, nos três tipos analisados, foi menor que $0,5 \%$, podendo-se concluir, portanto, que o erro é, praticamente, desprezível. Todavia, a existência de alguns diagnósticos, entre essas pequenas inconsistências, é bastante desabonadora como prova, por exemplo, a ocorrência de oito casos de varíola e alguns de esquizofrenia, em menores de 1 ano (Fernandes et al., 2000). Este estudo, entretanto, não avaliou se o diagnóstico declarado era o principal, se existia, de fato, ou mesmo se teria havido erro de codificação. Por outro lado, a análise quanto à confiabilidade do diagnóstico de doenças crônicas não-transmissíveis não foi realizada.

\section{Morbidade ambulatorial}

Trata-se do SIA/SUS, implantado no País em 1991. O Sistema é bastante criticado e fornece, para o gestor, mais dados quanto ao movimento de atendimentos e contas ambulatoriais do que informações epidemiológicas. De fato, não prevê um registro individual do paciente, o que impede a realização de estudos sobre procedência, fluxos de demanda, bem como a caracterização da clientela, em relação aos serviços consumidos (M ello Jorge et al., 2001).

\section{Agravos de notificação obrigatória}

O Sistema de Informações de Agravos de Notificação - SINAN - foi criado visando racionalizar o processo de coleta e transferência de dados, relacionados às doenças e agravos de notificação compulsória. Foi implantado a partir de 1993 e sua implementação não é completa no País. A experiência tem mostrado que os dados derivados deste sistema são bastante consistentes quanto à confiabilidade do ponto de vista qualitativo; entretanto, quantitativamente, isto é, quanto à cobertura, ainda deixa a desejar. É importante notar, entretanto, que os dados do SINAN, baseados na Lista Nacional de Agravos de Notificação Obrigatória, referem-se apenas a doenças transmissíveis. 


\section{Comentários}

Os numerosos trabalhos nacionais e internacionais citados, desde Graunt, apontam claramente para críticas feitas aos dados de mortalidade. Entretanto, mesmo com esse aspecto negativo, tais estatísticas, como já salientado, foram e continuam sendo um ótimo instrumental para análises epidemiológicas. Estudos clássicos na área da epidemiologia descritiva trouxeram grande contribuição, por exemplo, no caso de cânceres utilizando as estatísticas de mortalidade rotineiras, como os realizados por Stewart e colaboradores (1978), Kaplan (1985), MacM ahon (1962) e outros que fizeram pesquisas retrospectivas específicas sobre leucemia, utilizando atestados de óbito como ponto de partida. Não se pode deixar de mencionar os estudos de Doll \& Hill (1956) e de Haenszel e colaboradores (1962) sobre associação entre tumores sólidos de várias localizações e o hábito de fumar. Doll (1955), Knox e colaboradores (1968), em estudos de seguimento, com igual fonte, verificaram que câncer de pulmão estava associado ao trabalho em indústrias que utilizavam asbestos.

Vários outros trabalhos poderiam ser citados sobre usos das estatísticas de mortalidade por causas, mostrando possíveis associações. Entre esses, Seltser \& Sartwell (1965), M atanoski e colaboradores (1975), Court Brown \& Doll (1958), que compararam a mortalidade de médicos radiologistas com a de outras especialidades médicas, encontrando excesso de mortalidade por câncer nesse grupo profissional.

Foi a observação do que estava ocorrendo com a mortalidade por doenças cardiovasculares, com ênfase na cardiopatia aterosclerótica, desde as primeiras décadas do século 20 até meados dos anos 60, que levou vários epidemiologistas a declarar a ocorrência dessas causas como "a epidemia do século". Esses dados possibilitaram aos pesquisadores americanos descrever o declínio da tendência, a partir de 1968, o que foi observado também em vários países europeus (US N HI, 1979). Na mesma linha de raciocínio, as estatísticas de mortalidade rotineira permitiram mostrar o declínio observado em São Paulo, a partir da metade da década de 1970 (Lolio et al., 1986).

Ao comentar o declínio da mortalidade nos Estados Unidos (primeiro país onde isso foi descrito) na citada "Conferência sobre o Declínio", em 1979, foram discutidos possíveis fatores de erro nas estatísticas sobre mortes, con- cluindo-se que, mesmo existentes, não afetavam os resultados. Com isso, a tendência observada na mortalidade não era devida a artefatos estatísticos.

É importante assinalar que, como se comentou, embora as estatísticas de mortalidade ainda "padeçam" de al guns vieses, uma determinada tendência, quer seja ascendente, declinante ou estável, deve sempre ser aceita como verdadeira.

0 mais completo estudo até hoje realizado sobre doen ças cardíacas, acidentes vasculares cerebrais, fatores de risco e suas tendências em populações, é o M ÔNICA (M onitoring Cardiovascular Disease Study), produzido e supervisionado pela World $\mathrm{H}$ ealth Organization $\mathrm{e}$ que utilizou, entre outros dados, aqueles obtidos a partir das estatísticas de mortalidade rotineiras de 30 áreas de mais de 20 países. Uma das preocupações dos responsáveis pelo mesmo foi analisar a validade dos dados: One of the primary purposes of M ONICA was assess the validity of routine mortality data from death certificates. This is because there were concerns that trends in death rates from coronary heart diseases and stroke were artificialy affected by changes in reporting and coding practices, changes in the International Classification of Diseases and changes in diagnosis accuracy. In addition, death certificates coding may vary between countries leading to artificial differences in death rates, one explanation for the so-called "French Paradox" (WHO, 2003). Os resultados desse projeto trouxeram importantíssimas contribuições para avaliações epidemiológicas das doenças referidas e seus fatores de risco.

Pode-se dizer, assim, que as estatísticas de mortalidade por causas, ainda que não sejam totalmente exatas ou confiáveis, são utilíssimas para numerosos estudos epidemiológicos. Por outro lado, é bom ressaltar que os dados brasileiros de mortalidade, do ponto de vista qualitativo, são tão exatos ou fidedignos quanto aqueles oriundos de qualquer outro país de longa tradição na elaboração dessas estatísticas, como Estados Unidos, Canadá, Inglaterra, Suécia e outros países europeus.

0 que foi aqui descrito quanto à fidedignidade dos dados de mortalidade diz respeito apenas à declaração da causa básica, a qual nem sempre é feita de maneira correta pelos médicos. Por outro lado, as estatísticas podem apresentar falhas, mesmo quando a causa da morte é declarada corretamente, porque existe a possibilidade de erros na codificação da cau- 
sa, por conta de codificadores mal preparados. No Brasil, após a municipalização das ações de saúde, a codificação das causas de morte passou a ser feita nas secretarias municipais de saúde, o que fez com que a quantidade de codificadores crescesse assustadoramente, sendo que o Brasil é, hoje, talvez, o país com maior número de codificadores de causas de morte. Esse fato fez com que se tornasse difícil o seu treinamento e, particularmente, o acompanhamento e a supervisão necessários para o bom andamento dos trabalhos. Assim, corre-se o risco de ver deterioradas as estatísticas de mortalidade, caso não seja feita boa formação dos codificadores e supervisão, em nível municipal. A tentativa de sanar essa questão e uniformizar o trabal ho desenvolvido foi feita por meio de um programa para seleção eletrônica da causa básica da morte, o qual vem sendo utilizado regularmente através do programa Seletor da Causa Básica - SCB, desenvolvido pelo Centro Brasileiro de Classificação de Doenças em conjunto com o Datasus/M inistério da Saúde. Este, embora ainda com algumas falhas, representa a esperança de uma boa codificação da causa da morte, além de assegurar a comparabilidade dos seus dados, em todo o País.

\section{Colaboradores}

R Laurenti, M H P M ello Jorge SLD Gotlieb participaram igualmente de todas as etapas da elaboração do artigo.

\section{Referências bibliográficas}

Abramson JH, Sack MI \& Cahana E 1971. Death certificate data as an indication of the presence of certain common diseases at death. Journal of Chronicle Diseases 24:417-431

Barros FC, Victora CG, Teixeira AM \& Puerto M 1985. Mortalidade perinatal e infantil em Pelotas, Rio Grande do Sul: nossas estatísticas são confiáveis? Cadernos de Saúde Pública 1:348-358.

Breaux S\& Perez CA 1984. Pitfalls in the use of death certificates for assessing cause of death: A study of tonsil carcinoma patients. American Journal of Clinical Oncology 7:375-380.

Cabot RC 1912. Diagnostic pitfalls identified during a study of three thousand autopsies. Journal of the American M edical Association LIX:2295-2298.
O utro fator que pode afetar as estatísticas de mortalidade por causas, somente para estudos de tendências, é a introdução de novas revisões da Classificação Internacional de D oenças. Com o objetivo de sanar esse viés, a OM S e a Rede de Centros Colaboradores da OM S para a Classificação de Doenças publicam uma equivalência dos códigos entre as revisões, a qual deve ser usada nesses estudos, sempre que o período estudado englobar mais do que uma revisão. Um cuidado também a ser tomado para evitar distorção dos dados, na análise epidemiológica, refere-se à necessidade de selecioná-los segundo local de residência do falecido ou, em caso específico, segundo local de ocorrência. Além de todos esses aspectos, é preciso introduzir no currículo das escolas médicas (nos últimos anos e mesmo na residência médica) temas relacionados às estatísticas de mortalidade, seus usos e a importância do preenchimento do atestado de óbito e a maneira correta de fazêlo.

A excessiva multiplicação dos cursos de me dicina é bastante preocupante, visto que pode levar a uma situação em que, se os alunos estão correndo o risco de serem mal preparados nas artes de diagnosticar e tratar, certamente, não serão melhores quanto a declarar do que morreram seus pacientes.
Chio A, Magnani C, Oddenino E, Tolardo G \& Shiffer D 1992. Accuracy of death certificate diagnosis of amyotrophic lateral sclerosis. Journal of Epidemiology and Community H ealth 46:517-518.

Court Brown WN \& Doll R 1958. Expectation of life and mortality of cancer among British radiologists. British M edical Journal 2:181-187

Demers PA et al. 1992. Cancer identification using a tumor registry versus death certificates in occupational cohort studies in the United States. American Journal of Epidemiology 136:1323-1240.

Doll R \& Hill BA 1956. Lung cancer and other causes of death in relation to smoking. British M edical Journal 2:1071-1077.

Doll R 1955. M ortality from lung cancer in asbestos work- 
ers. British Journal of the Industrial M edicine 12:8187.

Fernandes W, Laurenti R, Buchalla CM \& Carré M L 2000. Accuracy of the information about diagnosis in the discharge in public hospitals in Brazil. Apresentado na Reunião de Diretores de Centros de Classificação de Doenças, em Colônia, Alemanha.

Garrucho $\mathrm{G}$ et al. 1990. Análisis de la concordancia de los dados recogidos en el certificado médico de defunción y el boletin estatistico de defunción. Revista Sanitaria de Higiene Pública: 64:63-72

Gittelsohn A \& Senning J 1979. Studies on the reliability of vital and health records: I-Comparison of cause of death and hospital record diagnosis. American Journal of Public Health: 69:680-689

Graunt J 1964. O bservations. Journal of the Institute of Actuaries 90:1-61

Haenszel W, Loveland DB \& Sirken M G 1962. Lung cancer mortality as related to residence and smoking history. I: White M ales. Journal National of Cancer Institute 28:947-953

Heasman M A \& Lipworth L 1966. Accuracy of certification of causes of death. Studies on M edical and Population Subjects № 20. HM SO, Londres.

Kagan A, Katsuk S, Sternby N \& Vanecek R 1967. Reliability of death certificates data on vascular lesions affecting the central nervous system. Bulletin WHO 37: 477-481

Kaplan HS 1985. An evolution and somatic and genetic hazards of the medical uses of radiation. American Journal Roentography 80:696-701.

Knox JF, Holmer S, Doll R \& Hill ID 1968. M ortality from lung cancer and others causes in workers in an asbestos textile factory. British Journal of the Industrial M edicine 25:293-298.

Laurenti R 1973. Causas múltiplas de morte. Tese de Livre Docência, Faculdade de Saúde Pública, USP, São Paulo.

Laurenti R, Aylin P \& Coleman M P 2000. Accuracy of statements of the cause of death on death certificates and the international comparability of mortality statistics. In M P Coleman \& P Aylin (eds.). Death certification and mortality statistics: an international perspective. Studies on M edical and Population Subjects no 64. The Stationery Office, Londres.

Laurenti R, M ello Jorge M H P \& Gotlieb SLD 2002. Estudo de mortalidade de mulheres de 10 a 49 anos - Projeto GPP - gravidez, parto e puerpério Relatório enviado ao M S/ Área Técnica de Saúde da M ulher). São Paulo.

Laurenti R, M ello Jorge M HP \& Gotlieb SLD 2003. The accuracy of medical statement of the cause of death in death certificate. Apresentado na Reunião de Diretores de Centros de Classificação de Doenças, em Colônia, Alemanha.

Lolio CA, Souza JM P \& Laurenti R 1986. Decline in cardiovascular diseases mortality in the city of São Paulo, Brazil. Revista de Saúde Pública 20(6):454-464.

M acM ahon B 1962. Pre-natal X-ray exposure and childhood cancer. Journal of National Cancer Institute 28:1173-1180.

M atanoski GM, Seltser R \& Sartwell PE 1975. The current mortality rate of radiologists and other physician specialties. American Journal of Epidemiology 101(3): 199-210.
M ello Jorge M H P, Gotlieb SLD \& Laurenti R 2001. A saúde no Brasil: análise do período 1996 a 1999. Organização Pan-Americana da Saúde, Braślia.

M ello Jorge M H P, Gotlieb SLD \& Laurenti R 2002. 0 sistema de informações sobre mortalidade: problemas e propostas para o seu enfrentamento. I - mortes por causas naturais. Revista Brasileira de Epidemiologia 5-2:197-201.

M inistério da Saúde: Datasus/ SIM , 2002. Disponível em $<$ <ww.datasus.gov.br>

M inistério da Saúde: Datasus/ SIH , 2002. Disponível em $<$ www.datasus.gov.br>

M oriyama IM 1979. Public uses of mortality data. In Proceedings of the meeting on socioeconomic determinants and consequences of mortality. El Colégio de M éxico, 19-25 June, 1979. United Nations and World Health Organization, New York-Geneva.

M oyer LA, Boyle CA \& Pollock DA 1989. Validity of death certificates for injury-related causes of death. American Journal of Epidemiology 130:1024-1032.

OM S 1995. Classificação Estatística Internacional de Doenças e Problemas Relacionados à Saúde (CID-10). Centro Brasileiro de Classificação de Doenças, São Paulo. (vol. l ell).

Pohlen K \& Emerson H 1942. Errors in clinical statement of causes of death. American Journal of Public $\mathrm{H}$ ealth 32:252-259.

Puffer RR \& Griffith GW 1968. Patterns of urban mortality. Report of the InterAmerican investigation of mortality. Pan-American Heath Organization, Washington, DC. (Scientific Publication, № 15)

REDE Interagencial de Informações para a Saúde - RIPSA 2002. Indicadores básicos de saúde no Brasil: conceitos e aplicações. Organização Pan-Americana da Saúde, Brasília.

Rothman KJ 1996. Lessons from John Graunt. Lancet 347:37-39

Rutqvist LE 1985. Validity of certified causes of death in breast carcinoma patients. Acta of Radiology Oncology 24:385-390.

Sanches AM 2003. Acurácia do preenchimento dos atestados de óbito com validação diagnóstica por meio das autópsias realizadas em hospital universitário do município de São Paulo. Tese de doutorado. Faculdade de Saúde Pública, USP.

Seltser R \& Sartwell PE 1965.The influence of occupational exposure to radiation on the mortality of American radiologists and other medical specialties. American Journal of Epidemiology 81(1):2-22.

Stewart A, Webb J \& Hewitt J 1978. A survey of childhood malignancies. British M edical Journal 1:1495-1500.

US D eptartment of Health, Education and Welfare. Public Health Services, NIH 1979. Proceedings of the conference on the decline in coronary heart disease mortality. Ed. Richard J. Havlick \& M anning Feinleib. NIH Publication № 76-1610.

World Health Organization 2003. M onica monograph and multimedia sourcebook. Ed. Hugh Tunstall - Pedol, Geneva.

Artigo apresentado em 9/3/2004

Aprovado em 15/8/2004

Versão final apresentada em 20/8/2004 